Review began 01/31/2022 Review ended 03/04/2022 Published 03/06/2022

(c) Copyright 2022

Ashish et al. This is an open access article distributed under the terms of the Creative Commons Attribution License CC-BY 4.0., which permits unrestricted use, distribution, and reproduction in any medium, provided the original author and source are credited.

\section{Importance of Early Next-Generation Sequencing in Microsatellite Unstable Colon Cancer With a High Tumor Mutation Burden}

\author{
Sethi Ashish ${ }^{1}$, Moses Raj ${ }^{2}$ \\ 1. Medical Oncology, Allegheny Health Network, Pittsburgh, USA 2. Hematology \& Oncology, Allegheny Health \\ Network, Pittsburgh, USA
}

Corresponding author: Sethi Ashish, sethiashish4@yahoo.com

\begin{abstract}
Colon cancer is one of the leading causes of cancer-related deaths. Microsatellite instability (MSI) or deficient mismatch repair proteins with a high tumor mutation burden (TMB) colon cancer are less responsive to chemotherapy. Targeted therapies based on early next-generation sequencing (NGS) in metastatic colon cancer can help significantly in overall prognosis. Here, we report a case of colon cancer that illustrated significant TMB and MSI and responded poorly to treatment due to delay in NGS testing.
\end{abstract}

Categories: Internal Medicine, Oncology

Keywords: next-generation sequencing (ngs), msi high, metastatic colorectal cancer, polo trial, olympia trial, brca gene mutation - predominantly brca2, keynote 177, colon cancer, microsatellite instability (msi), tumor mutation burden

\section{Introduction}

Microsatellite instability (MSI)-high or deficient mismatch repair (dMMR) proteins have been seen in many malignancies, including the gastrointestinal and genitourinary systems [1]. Colon and endometrial cancer, in particular, have been reported to have higher MSI prevalence [2]. Roughly 12-15\% of patients with colorectal cancer (CRC) and $4 \%$ of patients with metastatic colorectal cancer (mCRC) are classified as MSIhigh or dMMR type [3]. Next-generation sequencing (NGS) has been widely applied in CRC screening, diagnosis, and treatment in clinical settings [4]. New treatment regimens with immune checkpoint inhibitors (ICIs) or combination therapies based on molecular profiling for appropriate therapy have the potential to improve overall survival (OS) in aggressive colon cancer.

\section{Case Presentation}

We report the case of a 73-year-old male with metastatic colon cancer who had a delay in NGS testing due to surgery and declining performance status. The patient presented in the emergency room (ER) with complaints of abdominal pain, bloating, and weight loss. He had started to feel unwell about one year ago. His symptoms were associated with fatigue and decreased appetite. The patient also reported a weight loss of 25 pounds in three weeks. Computerized tomography (CT) of his abdomen showed an ileocecal mass with small bowel obstruction (SBO). Subsequently, the patient underwent an open right colectomy with end ileostomy. Intraoperative findings revealed diffuse carcinomatosis involving the anterior abdominal wall in the pelvis and abdominal mesentery. There was also a large mass occupying the cecum and obstructing the small bowel and the appendix with mild ascites. Surgical pathology revealed stage III or T4aN2bM0 invasive poorly differentiated adenocarcinoma with mucinous and signet ring cell differentiation (Figure 1).

Carcinoma was noted to invade the visceral peritoneum and appendix by direct extension. There was extensive lymphovascular invasion (LVI) and 17/19 lymph nodes were involved. Immunohistochemistry (IHC) testing showed loss of nuclear expression of MutL homolog 1 (MLH1) and mismatch repair PMS1 homolog 2 (PMS2) proteins (Figure 2). The patient had prolonged recovery time post-surgery which led to a delayed referral to the oncologist for further management. During the colorectal multidisciplinary team meeting, adjuvant chemotherapy was suggested in view of metastatic disease and diffused carcinomatosis. The plan was to complete eight cycles of a combination of folinic acid, fluorouracil, and oxaliplatin (FOLFOX) chemotherapy for over six months prior to the result of NGS. After receiving the first cycle of FOLFOX, the treatment plan was switched to pembrolizumab based on somatic NGS testing (Tables 1, 2). Molecular testing or NGS revealed a high TMB of 62.1 mutations per megabase (m/MB) and MSI (Table 3). Unfortunately, the patient only completed one cycle of pembrolizumab and died three weeks later. 


\section{Cureus}

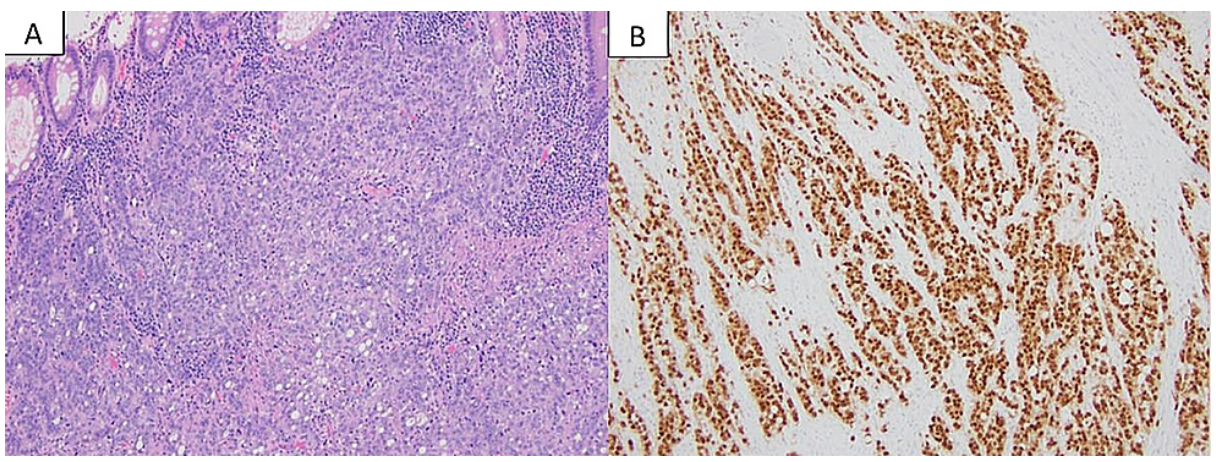

FIGURE 1: Surgical pathology: colonic adenocarcinoma.

Surgical resection reveals a poorly differentiated adenocarcinoma with infiltrating lymphocytes and features of dMMR carcinoma ( $A, H \& E$ stain). The tumor cells are strongly positive for CDX2 (B, immunostain), supporting a diagnosis of colonic adenocarcinoma.

Permission was taken from Leo et al. [5].

dMMR: deficient mismatch repair; H\&E: hematoxylin and eosin

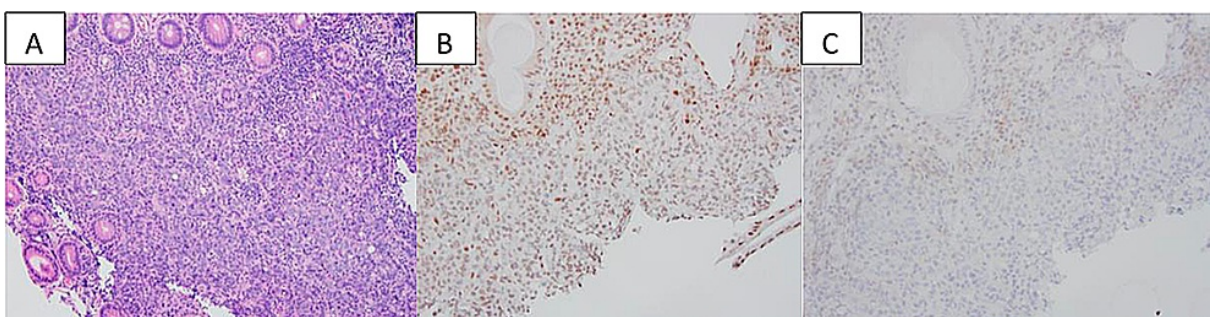

FIGURE 2: Immunohistochemical results of the biopsied colonic adenocarcinoma.

Biopsy of the cecal mass reveals sheet of cancerous cells (A, H\&E stain). The cells show loss of nuclear immunoreactivity for MLH1 (B, immunostain) and PMS2 (C, immunostain) in cancer. The overall features support a diagnosis of colonic adenocarcinoma with dMMR.

Permission was taken from Leo et al. [5].

H\&E: hematoxylin and eosin; MLH1: MutL homolog 1; PMS2: mismatch repair PMS1 homolog 2; dMMR: deficient mismatch repair

\begin{tabular}{|c|c|c|}
\hline Somatic/Gene type & Mutation effect & Variant allele fraction: Percentage (\%) \\
\hline BRCA2 & p.N1784fs frameshift-LOF & 13.7 \\
\hline PIKЗСA & p.R88Q missense variant (exon1)-GOF & 8.3 \\
\hline KRAS & p.G12D missense variant (exon 2)-GOF & 7.3 \\
\hline PIКЗСА & p.EK42K missense variant (exon 9)-GOF & 7.2 \\
\hline
\end{tabular}

TABLE 1: Genomic variants: Somatic, potentially actionable.

GOF: gain of function; LOF: loss of function; BRCA2: breast cancer gene 2; PIK3CA: phosphatidylinositol-4,5-bisphosphate 3-kinase catalytic subunit alpha; KRAS: Kirsten rat sarcoma viral oncogene homolog 


\section{Cureus}

\begin{tabular}{|c|c|c|}
\hline Somatic/Gene type & Mutation effect & Variant allele fraction: Percentage (\%) \\
\hline MSH6 & p.T1219I missense variant-LOF & 7.7 \\
\hline MSH3 & p.K383fs frameshift-LOF & 7.6 \\
\hline$A S X L 1$ & p.F408fs frameshift-LOF & 7.5 \\
\hline CTCF & p.H517fs frameshift-LOF & 6.4 \\
\hline CUL3 & p.D432fs frameshift-LOF & 6.4 \\
\hline PTCH1 & p.S1137fs frameshift-LOF & 5.4 \\
\hline TP53 & p.G245S missense variant-LOF & 5.3 \\
\hline RB1 & c.539+1G>A splice region variant-LOF & 5.1 \\
\hline FLCN & p.H429fs frameshift-LOF & 22.8 \\
\hline$B C O R$ & p.R1164 stop gain-LOF & 20.5 \\
\hline CTNNB1 & p.T41A missense variant-GOF & 20.1 \\
\hline MSH6 & p.F1088fs frameshift-LOF & 14.7 \\
\hline MSH6 & p.F1088fs frameshift-LOF & 12.3 \\
\hline CHD2 & p.K139fs frameshift-LOF & 10.4 \\
\hline SETD2 & p.N1396fs frameshift-LOF & 9.5 \\
\hline ARID2 & p.N1778fs frameshift-LOF & 9.5 \\
\hline CUX1 & p.T611fs frameshift-LOF & 8.9 \\
\hline PPP6C & p.F253fs frameshift-LOF & 8.9 \\
\hline RASA1 & p.P139fs frameshift-LOF & 8.8 \\
\hline$F B X W 7$ & p.R505H missense variant-LOF & 8.7 \\
\hline PPM1D & p.R572 stop gain-GOF & 8.6 \\
\hline TP53 & p.E204K missense variant-LOF & 8.6 \\
\hline CREBBP & p.R1360 stop gain-LOF & 8.3 \\
\hline ARD1A & p.D1850ts frameshift-LOF & 8.2 \\
\hline$A P C$ & p.N1792fs frameshift-LOF & 8.0 \\
\hline
\end{tabular}

\section{TABLE 2: Genomic variants: Somatic, biologically relevant.}

MSH6: MutS homolog 6; MSH3: MutS homolog 3; ASXL1: additional sex combs transcriptional regulator 1; CCTF-11: zinc finger protein or CCCTC-binding factor; CUL3: Cullin based E3ligase; PTCH1: protein patched homolog 1; TP53: tumor protein p53; RB1: retinoblastoma protein; FLCN: folliculin; BCOR: BCL6 corepressor; CTNNB1: catenin beta 1; CHD2: chromodomain helicase DNA binding protein 2; SETD2: SET domain containing 2, histone lysine methyltransferase; ARID2: AT-rich interaction domain 2; CUX1: Cut like homeobox 1; PPP6: protein phosphatase 6 catalytic subunit; RASA1: RAS P21 protein activator 1; FBXW7-F: Box and WD repeat domain containing 7; PPM1D: protein phosphatase, $\mathrm{Mg}^{2+} / \mathrm{Mn}^{2+}$ dependent 1D; CREBPP: cyclic adenosine monophosphate response element binding protein; ARID1A: AT-rich interactive domain-containing protein 1A; APC: adenomatous polyposis coli 


\section{Cureus}

Tumor mutation burden

$62.1 \mathrm{~m} / \mathrm{MB}$
Microsatellite instability status

High

TABLE 3: Tumor mutation burden/MSI-high.

m/MB: mutation per megabase; MSI: microsatellite instability

\section{Discussion}

Rectal bleeding or hematochezia, microcytic hypochromic anemia, altered bowel movements, abdominal pain, and weight loss are common initial clinical presentations associated with metastatic colon cancer. Lymph nodes, liver, and peritoneum are common sites of metastasis in colon cancer. NGS is a remarkable way to analyze multiple genes to identify targetable mutations. For example, a vendor like Tempus would look at 648 genes from tissue biopsy and 105 genes with liquid or plasma bases assay. For some malignancies, such as CRC, MSI, or dMMR phenotype may be a predictive factor for high TMB and respond to ICIs.

MSI-high or dMMR subtype can be detected by polymerase chain reaction (PCR) or IHC. Chemotherapies such as FOLFOX, capecitabine, bevacizumab, and ramucirumab are used as a single agent or in combination as adjuvant or palliative treatment depending upon the stage of colon cancer. Presently, ICIs such as programmed cell death protein inhibitors have been approved for MSI-high CRC first line. Phase III KEYNOTE-177 trial is a classic example of high response rates with immunotherapy compared to chemotherapy [6]. Pembrolizumab monotherapy demonstrated a statistically significant improvement in progression-free survival (PFS) compared with chemotherapy. Median PFS in the trial illustrated 16.5 months versus 8.2 months, with a hazard ratio (HR) of 0.60 and $95 \%$ confidence interval (CI) of $0.45-0.80$; two-year PFS rate was $48.3 \%$ versus $33.1 \%$. The advantage of using pembrolizumab or ICI was consistent except for those having RAS-mutated tumors ( $\mathrm{HR}=1.19,95 \% \mathrm{CI}=0.68-2.07$ ) [6]. Nevertheless, despite substantial clinical benefits with ICIs, $40-50 \%$ of MSI or dMMR MCRC cases manifest resistance to ICI monotherapy [6]. TMB is a useful biomarker for the consideration of immunotherapy in several cancer types revealed by NGS. It denotes the number of mutations carried by malignant cells. High TMB in cancers may be linked with environmental factors such as chronic exposure to ultraviolet light for melanoma or tobacco exposure for lung, head, and neck cancers [7].

mCRC includes biomarkers such as B-Raf, neurotrophic tyrosine receptor kinase, KRAS G12C, and human epidermal growth factor receptor 2 are actionable as well as positive and negative predictors of the overall response. As our patient harbored a high allele fraction of somatic BRCA2 mutation, consideration of polyadenosine diphosphate-ribose polymerase (PARP) inhibitor such as olaparib or talazoparib to prevent progression in MSI-high colon cancer could have led to a positive outcome based on the Olympia trial and the pancreas cancer olaparib ongoing (POLO) trial. The Olympia trial illustrated that one year of adjuvant olaparib, PARP inhibitors can decrease recurrence risk and prevent progression to metastatic disease in patients with high-risk early breast cancer and BRCA1 or 2 pathogenic variants, with low-grade toxicity index [8]. The POLO trial also showed that maintenance olaparib provided a significant PFS benefit to patients with a germline BRCA mutation and metastatic pancreatic cancer that had not progressed with platinum-based chemotherapy [9].

\section{Conclusions}

Early referrals to oncologists and genomic analysis can improve survival for mCRC patients. Unfortunately, our patient presented at a very late stage with declining performance status resulting in early mortality. In this patient, with timely reporting, the addition of BRCA1 inhibitors such as PARP inhibitors with other mutations could have yielded a better outcome. Moreover, as noted in the KEYNOTE 177 trial, MSI-high status improves the likelihood of response to ICIs.

\section{Additional Information \\ Disclosures}

Human subjects: Consent was obtained or waived by all participants in this study. Conflicts of interest: In compliance with the ICMJE uniform disclosure form, all authors declare the following: Payment/services info: All authors have declared that no financial support was received from any organization for the submitted work. Financial relationships: All authors have declared that they have no financial relationships at present or within the previous three years with any organizations that might have an interest in the submitted work. Other relationships: All authors have declared that there are no other relationships or activities that could appear to have influenced the submitted work. 


\section{Cureus}

\section{Acknowledgements}

The completion of this undertaking could not have been possible without the participation and assistance of so many people whose names may not all be enumerated. Their contributions are sincerely appreciated and gratefully acknowledged. To all relatives, friends, and others who in one way or another shared their support, either morally, financially, or physically, thank you.

\section{References}

1. Trullas A, Delgado J, Genazzani A, et al.: The EMA assessment of pembrolizumab as monotherapy for the first-line treatment of adult patients with metastatic microsatellite instability-high or mismatch repair deficient colorectal cancer. ESMO Open. 2021, 6:100145. 10.1016/j.esmoop.2021.100145

2. Le DT, Durham JN, Smith KN, et al.: Mismatch repair deficiency predicts response of solid tumors to PD-1 blockade. Science. 2017, 357:409-13. 10.1126/science.aan6733

3. Baretti M, Le DT: DNA mismatch repair in cancer. Pharmacol Ther. 2018, 189:45-62. 10.1016/j.pharmthera.2018.04.004

4. Xiao J, Li W, Huang Y, et al.: A next-generation sequencing-based strategy combining microsatellite instability and tumor mutation burden for comprehensive molecular diagnosis of advanced colorectal cancer. BMC Cancer. 2021, 21:282. 10.1186/s12885-021-07942-1

5. De Leo EK, Shah CP, Grajo JR, Liu X, Parekh H: Extramedullary hematopoiesis in mismatch repair deficient colon cancer patient on adjuvant chemotherapy. Cureus. 2021, 13:e12899. 10.7759/cureus.12899

6. Cohen R, Colle R, Pudlarz T, Heran M, Duval A, Svrcek M, André T: Immune checkpoint inhibition in metastatic colorectal cancer harboring microsatellite instability or mismatch repair deficiency. Cancers (Basel). 2021, 13:10.3390/cancers13051149

7. Sha D, Jin Z, Budczies J, Kluck K, Stenzinger A, Sinicrope FA: Tumor mutational burden as a predictive biomarker in solid tumors. Cancer Discov. 2020, 10:1808-25. 10.1158/2159-8290.CD-20-0522

8. Tutt AN, Garber JE, Kaufman B, et al.: Adjuvant olaparib for patients with BRCA1- or BRCA2-mutated breast cancer. N Engl J Med. 2021, 384:2394-405. 10.1056/NEJMoa2105215

9. Golan T, Hammel P, Reni M, et al.: Maintenance olaparib for germline BRCA-mutated metastatic pancreatic cancer. N Engl J Med. 2019, 381:317-27. 10.1056/NEJMoa1903387 